\title{
Plasmaféresis como opción terapéutica en infección por COVID-19
}

\section{Plasmapheresis as a therapeutic option in COVID-19 infection}

\author{
Jorge-Eduardo Rico-Fontalvo ${ }^{1}$; Nehomar Pájaro-Galvis²; Víctor Leal-Martínez²; Rodrigo Daza-Arnedo ${ }^{3}$; Emilio Rey-Vela ; \\ Luis-Gabriel Salgado-Montiel ${ }^{3}$; María-Carolina Monterrosa-Robles ${ }^{3}$; Alonso Pomares-Lara ${ }^{5}$
}

Forma de citar: Rico-Fontalvo JE, Pájaro-Galvis N, Leal-Martínez V, Daza-Arnedo R, Rey-Vela E, Salgado-Montiel LG, et al. Plasmaféresis como opción terapéutica en infección por Covid-19. Salud UIS. 2020; 52(3): 311-318. doi: http://dx.doi.org/10.18273/revsal.v52n3-2020011 (c) (i)

\section{Resumen}

Introducción: El COVID-19, es una neumonía ocasionada por el nuevo coronavirus SARS-CoV-2, que se puede presentar con cuadros severos, distrés respiratorio agudo (SDRA), disfunción orgánica múltiple y muerte; como consecuencia de una respuesta inflamatoria alterada denominada "tormenta de citoquinas". La plasmaféresis se propone como una estrategia de tratamiento prometedora para el manejo de este tipo de complicaciones. Objetivo: nuestro objetivo principal es mostrar toda la bibliografía disponible, referente a la utilidad de la plasmaféresis en el manejo de la tormenta de citoquinas en pacientes con COVID-19 grave; evaluar su posible beneficio y proponer realizar nuevos ensayos clínicos que avalen el uso rutinario de esta terapia. Metodología: Se realizó una búsqueda avanzada con los términos DeSC “Infecciones por Coronavirus; SARS-CoV; Plasmaféresis; Recambio plasmático; Disfunción orgánica múltiple; Sepsis; Síndrome de respuesta inflamatoria sistémica; Lesión renal aguda". A través de los motores de búsqueda Clinical Key, Embase, PubMed y Ovid, obteniendo un total de 156 resultados, entre artículos originales, reportes de casos, series de casos y revisiones de la literatura, se seleccionaron un total de 54 artículos que fueron utilizados para la elaboración de la presente revisión de la literatura. Conclusiones: La terapia de recambio plasmático se podría utilizar como tratamiento complementario, con el objetivo de reducir carga inflamatoria y viral, reduciendo así el daño de órgano blanco. Sin embargo, hace falta la realización de ensayos clínicos controlados y con buenos diseños metodológicos, que nos ayuden a demostrar la efectividad de este tipo de terapias en pacientes con COVID-19 grave.

Palabras clave: Infecciones por Coronavirus; SARS-CoV; Plasmaféresis; Recambio plasmático; Disfunción orgánica múltiple; Sepsis; Síndrome de respuesta inflamatoria sistémica; Lesión renal aguda.

\footnotetext{
1. Clínica SOMA, Medellín; Antioquia

2. Universidad del Sinú, Cartagena, Bolívar

3. Nuevo Hospital Bocagrande, Cartagena, Bolívar

4. Hospital universitario Samaritana, Bogotá, Cundinamarca

5. Clínica San José de Torices, Cartagena, Bolívar

Correspondencia: Nehomar Pájaro Galvis. Dirección: Transversal 54 41-117 U. Sinú, Cartagena. Teléfono: +57 3008233570. Correo electrónico: neho94@hotmail.com
} 


\begin{abstract}
Introduction: COVID-19 is a pneumonia caused by the new SARS-CoV-2 coronavirus, which can present with severe symptoms, acute respiratory distress (ARDS), multiple organ dysfunction and death; as a consequence of a dysregulated inflammatory response called "cytokine storm". Plasmapheresis is proposed as a promising treatment strategy for the management of this type of complications. Objective: our main objective is to show all the available bibliography, referring to the utility of plasmapheresis in the management of cytokine storm in patients with severe COVID-19; evaluate its possible benefit and propose new clinical trials to support the routine use of this therapy. Methodology: An advanced search was performed with the DeSC terms "Coronavirus Infections; SARS-CoV; Plasmapheresis; Plasma exchange; Multiple organic dysfunction; Sepsis; Systemic inflammatory response syndrome; Acute kidney injury ". Through the search engines Clinical Key, Embase, PubMed and Ovid, obtaining a total of 156 results, among original articles, case reports, case series and literature reviews, a total of 54 articles were selected and used for the preparation of this literature review. Conclusions: Plasma replacement therapy could be used as a complementary treatment, with the aim of reducing inflammatory and viral loads, thus reducing target organ damage. However, it is necessary to carry out controlled clinical trials with good methodological designs that help us demonstrate the effectiveness of this type of therapy in patients with severe COVID-19.
\end{abstract}

Keywords: Coronavirus Infections; SARS-CoV; Plasmapheresis; Plasma exchange; Multiple organ failure; Sepsis; Systemic Inflammatory Response Syndrome; Acute Kidney Injury.

\section{Introducción}

La enfermedad por COVID-19, se trata de una neumonía ocasionada por el nuevo coronavirus SARS-CoV-2, cuyo curso clínico es muy heterogéneo, abarcando desde presentaciones leves, hasta pacientes con cuadros severos, desarrollo de síndrome de distrés respiratorio agudo (SDRA), disfunción orgánica múltiple y muerte. Estas complicaciones son explicadas en su mayoría, por la respuesta inflamatoria desregulada como consecuencia de una tormenta de citoquinas proinflamatorias, que desencadenan daño de órgano blanco, activación de factores procoagulantes y oclusión de la microcirculación. A partir de esta premisa, existen alternativas de tratamiento para reducir la expresión de citoquinas proinflamatorias, dentro de las que se incluye la plasmaféresis y la terapia de recambio plasmático; que, si bien es cierto, su evidencia científica en COVID-19 es escasa, han demostrado beneficio en otros escenarios similares como la Sepsis.

\section{Objetivos}

Teniendo en cuenta la crisis de salud mundial ocasionada por la pandemia COVID-19, nuestro objetivo principal es mostrar la toda la bibliografía disponible, con referencia a la utilidad de la plasmaféresis en el manejo de la tormenta de citoquinas en los pacientes con COVID-19 y manifestaciones graves; evaluar su posible beneficio y proponer la realización de nuevos ensayos clínicos que avalen el uso rutinario de esta terapia.

\section{Metodología}

Se realizó una búsqueda avanzada con los términos DeSC y MeSH: "Infecciones por Coronavirus; SARS-CoV; Plasmaféresis; Recambio plasmático; Disfunción orgánica multiple; Sepsis; Síndrome de respuesta inflamatoria sistémica; Lesión renal aguda". Se utilizaron los motores de búsqueda Clinical Key, Embase, PubMed y Ovid, obteniendo un total de resultados, entre artículos originales, reportes de casos, series de casos y revisiones de la literatura, se seleccionaron un total de 54 artículos que fueron utilizados para la elaboración de la presente revisión de la literatura.

\section{COVID-19}

En diciembre de 2019, surge en la provincia de WuhanChina, una serie de casos de pacientes con síntomas respiratorios secundarios a una neumonía de origen desconocido, con un factor epidemiológico en común, originado en el mercado de mariscos Huanan en el sur de esta ciudad ${ }^{1}$ y que se expandió rápidamente a la mayor parte del país. En enero de 2020, se logró mediante pruebas de secuenciación genética el aislamiento del microorganismo causal, un virus de ARN monocatenario que provisionalmente se conoció como "2019 novel coronavirus" por su parecido genético con el SARS-CoV aislado en 2002 en la provincia de Canton, también en China ${ }^{2}$. En febrero 
de 2020 la OMS declara la neumonía por COVID-19 como una enfermedad emergente, con alto impacto en la salud pública a nivel mundial ${ }^{3}$, y para el 11 de marzo de 2020, luego que la enfermedad se extendiera a nivel global, fue declarada como pandemia ${ }^{4}$. Continuó con un crecimiento exponencial de los casos y hasta la fecha del 29 de junio de 2020. Se han reportado 10.021.401 casos y 499.913 muertes en todo el mundo; en América el total de 5.042.486 casos y 244.791 muertes $^{5}$ y en Colombia 91.769 casos confirmados, con un total de 3.106 muertes, comprometiendo 630 de los 1122 municipios de todo el país ${ }^{6}$.

El SARS-CoV-2 es un virus envuelto, compuesto de ARN monocatenario ${ }^{7}$, que pertenece al orden Nidovirales, familia Coronaviridae, subfamilia Coronavirinae, genero Betacoronavirus, siendo estos, los únicos capaces de infectar a los seres humanos; pues los otros géneros (Alfacoronavirus, gammacoronavirus y deltacoronavirus) solo afectan a animales ${ }^{8}$. Su característica estructural principal es la presencia de proyecciones lineales espiculadas en su envoltura, que simulan una corona solar, lo que le da el nombre de coronavirus 9 . Cuenta con la proteína $\mathrm{N}$, que, junto a su $\mathrm{ARN}$, forman la nucleocápside; la proteína de envoltura; la proteína S o "spike" que se une al receptor de la célula huésped, y la proteína $\mathrm{M}$, también denominada proteína de membrana, que aumenta la respuesta inmune y el ensamblaje del virus ${ }^{9}$.

El hospedero natural del coronavirus son los animales, en su mayoría murciélagos o roedores, teniendo como huésped intermedio a los mamíferos; y en el caso del SARS-CoV-2, el pangolín ${ }^{10}$. Esto generó en un principio, la teoría inicial de ser una antropozoonosis, con una alta tasa de mutación que permitió su paso de especie a especie, sin embargo, en la actualidad el contagio de persona a persona constituye la principal vía de transmisión ${ }^{11}$. La enfermedad severa en seres humanos es producida principalmente por 3 especies: MERS-CoV, SARS-CoV y SARS-CoV-2, sin embargo, su curso clínico es muy heterogéneo. Se ha documentado una amplia variedad de manifestaciones clínicas que van desde individuos asintomáticos, cuadros respiratorios leves con síntomas generales como fiebre y malestar general, hasta un compromiso pulmonar severo con neumonía multilobar, que progresa a síndrome de dificultad respiratoria grave y la muerte ${ }^{12}$. Además, se puede presentar con manifestaciones extrapulmonares, dentro de las que predominan los síntomas gastrointestinales en el $26 \%$ de los casos, dados por disgeusia, vómitos y diarrea ${ }^{13}$.
El diagnóstico del virus se realiza por pruebas moleculares con aislamiento del material genético viral mediante reacción en cadena de la polimerasa (RT$\mathrm{PCR})^{14}$, que identifica tres grupos de genes: un gen que es para la transcriptasa reversa dependiente de RNA y 2 genes estructurales que codifican para la proteína $\mathrm{N} \mathrm{y}$ para la proteína E, respectivamente ${ }^{15}$. Dichas pruebas poseen una sensibilidad y especificidad que varían dependiendo del espécimen utilizado para la muestra, siendo mayor en el lavado broncoalveolar alcanzando una sensibilidad de un $93 \%{ }^{16}$.

El abordaje terapéutico inicial está basado en garantizar medidas de soporte, pues no existe hasta este momento un tratamiento curativo; además de que la evidencia científica es poca, lo cual conlleva a que la práctica clínica se base en conductas terapéuticas experimentales, enfocándose en la protección de vía aérea como manejo inicial de síndrome de dificultad respiratoria; sin dejar de lado, medidas dirigidas hacia el control de la respuesta inflamatoria sistémica generada en el marco de la enfermedad y la tormenta de citoquinas ${ }^{17}$.

\section{De la fisiopatologia al abordaje terapeutico: tormenta de citocinas}

Los coronavirus, clasificados filogenéticamente como virus de ARN monocatenario de sentido positivo, son los virus de ARN más grande conocidos con un genoma de aproximadamente $31 \mathrm{kB}^{18}$. La unión al receptor de enzima convertidora de angiotensina, ubicado en la superficie celular de diversos órganos del cuerpo, principalmente a nivel pulmonar, media la entrada del virus a la célula por la interacción con la proteína Spike en la envoltura viral, liberándose posteriormente el genoma viral en el citoplasma para su replicación y formación de nuevas partículas virales que llevan a la infección de células vecinas ${ }^{19}$.

Con la replicación del virus se exponen numerosos antígenos virales que finalmente conducen a la activación del sistema inmune innato y adaptativo; y la participación de linfocitos $\mathrm{B}$ y $\mathrm{T}$ citotóxicos lleva a una respuesta inmune masiva que se conoce

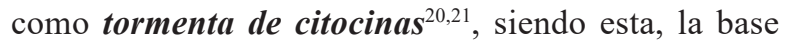
fisiopatológica del síndrome de dificultad respiratoria, falla multiorgánica y finalmente la muerte ${ }^{22,23}$. Este fenómeno inmunológico también ha caracterizado numerosas patologías, dentro de las que se destacan la infección por el virus de la gripe aviar ${ }^{24} \mathrm{y}$ la infección por las otras especies de coronavirus, SARS-CoV y MERS-CoV ${ }^{25}$. 
Durante la progresión de la neumonía por COVID-19, es bien conocido la presencia de linfopenia como marcador de gravedad en el desarrollo de la enfermedad ${ }^{26}$, con un numero notablemente disminuido de linfocitos TCD4, células TCD8, células B y células $\mathrm{NK}^{27}$, evidenciando también un aumento del número absoluto de neutrófilos durante la fase grave de la enfermedad ${ }^{28}$. El aumento del número de neutrófilos explica la producción elevada de citocinas proinflamatorias como IL-6, IL-1, FNT $\alpha$, e IL-8 y el recuento reducido de linfocitos conlleva a la disminución en el control natural de la enfermedad viral por la incapacidad de las células T citolíticas para lisar las células infectadas ${ }^{29}$.

La activación de la vía de la IL-6 mediante sus dos vías de señalización, Cis y Trans, genera una retroalimentación positiva del sistema de inflamosoma NLRP3, un complejo multiproteico que convierte a la procaspasa 1 en caspasa 1 activa, quien a su vez convierte la pro IL-1B en IL-1B activa prolongando de esta manera la cascada inflamatoria que perpetua la tormenta de citocinas en el desarrollo de la enfermedad moderada-grave, siendo esta condición una de las principales causas de muerte en pacientes con enfermedad por COVID-19 22,30-32.

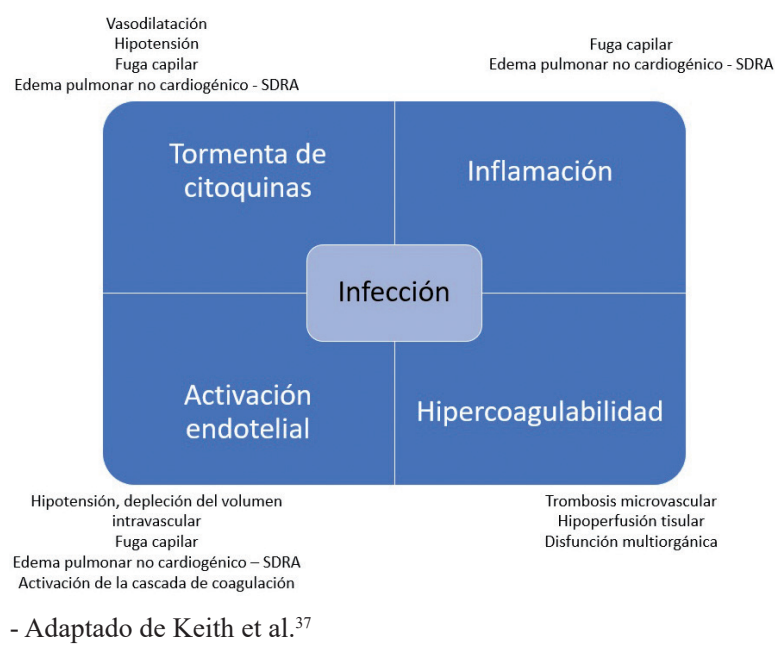

Figura 1. Resumen las consecuencias fisiopatológicas de la tormenta de citoquinas, la activación endotelial y el trastorno de hipercoagulabilidad.

\section{Plasmaferesis y terapia de recambio plasmatico como nuevo blanco terapeutico en Covid-19}

El brote por el nuevo coronavirus (SAR-CoV-2) se ha constituido un problema de salud pública a nivel mundial, debido a las cifras de contagio y mortalidad tan alarmantes en tan poco tiempo. Actualmente, no existen tratamientos curativos para la infección por COVID-19y las estrategias actuales de manejo se centran en medidas de soporte, el control de infecciones concomitantes y terapias experimentales que han mostrado utilidad en enfermedades con una base fisiopatológica común. Dentro de estas últimas destacan las terapias dirigidas contra la respuesta inflamatoria sistémica generada por el sistema inmune frente a el virus como el plasma convaleciente que contiene anticuerpos protectores, donados de los sobrevivientes de la infección por COVID-19, es un tratamiento prometedor y seguro ${ }^{33}$.

El intercambio terapéutico con plasma, una terapia no del todo novedosa, ya que se ha empleado en varios escenarios con éxito como el tratamiento de infecciones graves como la influenza A HIN1 2009, sepsis y falla multiorgánica ${ }^{34-38}$. Se ha propuesto como blanco terapéutico para el manejo de infección grave por COVID-19 mostrando una tasa de efectividad elevada en algunos reportes de $\operatorname{casos}^{39-42}$.

Durante el procedimiento de plasmaféresis se extrae la sangre del paciente y ésta es separada, diferenciando las partes formes del plasma, para permitir su flujo a lo largo de columnas con materiales adsorbentes que generen la eliminación de solutos específicos para posteriormente reinfudirlo al paciente ${ }^{32,43}$. En la terapia de recambio plasmático, también se separan las partes formes del plasma para su purificación, sin embargo, a diferencia de la plasmaféresis, al momento de la reinfusion el plasma es reemplazado por plasma de donante o por albúmina ${ }^{32,44}$. En la Figura 1 se resumen las consecuencias fisiopatológicas de la tormenta de citoquinas, la activación endotelial y el trastorno de hipercoagulabilidad, como posibles blancos de la terapia de recambio plasmático y la plasmaféresis.

En pacientes con sepsis y disfunción orgánica múltiple; la terapia plasmática podría mejorar la función del órgano blanco, al eliminar los mediadores inflamatorios y antifibrinolíticos y al reponer las proteínas anticoagulantes para restablecer la hemostasia ${ }^{45}$. Las pautas de la sociedad estadounidense de aféresis (ASFA) publicadas en 2019, designan la sepsis como categoría 3 (no se establece el papel óptimo de la terapia de aféresis; la toma de decisiones debe ser individualizada) y recomendación de grado $2 \mathrm{~b}$ (recomendación débil, moderada calidad de evidencia) para terapia de recambio plasmático ${ }^{46}$.

En el contexto de enfermedades virales, se ha documentado que a través de la plasmaféresis se podría eliminar ARN viral que induce la respuesta de 
citoquinas. Existe un informe que respalda el beneficio de la plasmaféresis de doble filtración para reducir el ARN viral para el virus de la hepatitis C (VHC), las cuales son suficientemente grandes (diámetro aproximado de 55-60 $\mathrm{nm}$ ) para atravesar la membrana y eliminarse. Esto resultó ser beneficioso logrando la regulación de la carga viral incluso entre pacientes que recayeron después de una terapia previa de inducción con interferón $\beta^{47}$. Similar a la plasmaféresis de doble filtración, durante el brote de H7N9, algunos módulos de purificación de sangre como el intercambio de plasma, la absorción de plasma y/o el hemo/plasma la filtración se ha utilizado con éxito para la eliminación de citocinas y quimiocinas ${ }^{48,49}$.

De manera similar, el SARS-CoV-2, tiene un diámetro de 60-140 nm, siendo lo suficientemente grande como para ser eliminado con DFPP, pudiendo ser beneficioso a la hora de usar esta terapia en pacientes críticos con insuficiencia respiratoria que no responde a tratamientos complementarios. Recientemente la FDA en Estados Unidos ha aprobado el uso de la exención de dispositivo de investigación (IDE) para toraymyxin (PMX) en el tratamiento de pacientes con COVID-19 que sufren shock séptico ${ }^{50}$.

La evidencia a favor del uso de plasmaféresis y terapia de recambio plasmático para COVID-19 es limitada y se basa en pequeños reportes de casos a lo largo de países como Japón, China y Estados Unidos con éxito terapéutico sobre todo en aquellos pacientes con compromiso de sistema nervioso central. Dogan, et al. reportaron una serie de casos, que incluyó 6 pacientes con COVID-19 grave, en ventilación mecánica por SDRA y compromiso de SNC (medido por LCR y Resonancia nuclear magnética de cerebro). Encontraron una mejoría de laboratorio notable después de la plasmaféresis, se observando disminución en los niveles de ferritina sérica en pacientes y una pronta mejoría clínica. Además, los hallazgos de MRI fueron reversibles en los tres pacientes a partir del control de MRI de la primera semana ${ }^{41}$. Estudios reportan su uso en pacientes con poca respuesta a tratamiento convencional y en un régimen terapéutico, que incluye como primer paso la reducción de virulencia con terapia antirretroviral, como segundo paso la reducción de la producción de citocinas y quimiocinas con terapia con corticoides o tocilizumab y, finalmente, la eliminación de citocinas circulantes con plasmaféresis obteniendo buenos resultados y disminución en el manejo de pacientes con insuficiencia respiratoria aguda ${ }^{51}$, un protocolo similar al utilizado en infecciones respiratorias como infección por virus de influenza ${ }^{49,52}$.
Recomendaciones del consenso colombiano de expertos sobre recomendaciones informadas en la evidencia para la prevención, diagnóstico y manejo de la lesión renal aguda por sars-cov-2/Covid-19.

En el consenso colombiano para la prevención, diagnóstico y manejo de la lesión renal aguda por SARS-CoV-2, se genera el siguiente interrogante: ¿Cuáles terapias extracorpóreas para aclaramiento de citoquinas disponible en Colombia podrían tener beneficio como parte del manejo de la tormenta de citoquinas asociada a COVID 19?; y se describen distintas modalidades de terapia extracorpórea orientadas a eliminar los factores inflamatorios, lograr estabilización hemodinámica, reducción de lactato y prevenir daño de órganos en pacientes con enfermedad severa; que incluyen: hemoperfusión directa usando un sorbente neutro-macroporoso, adsorción de plasma en una resina después de la separación del plasma de la sangre completa, TRRC con filtros de fibra hueca con propiedades de adsorción, TRRC de dosis alta con membranas de corte medio o corte alto, la terapia de recambio plasmático y la plasmaféresis ${ }^{53}$.

El intercambio de plasma elimina toxinas de peso molecular bajo a grande, además, tiene un potencial efecto en anomalías de coagulación ${ }^{54}$. Algunos expertos sugieren dosis de tratamiento entre 1.5 y 2 veces el volumen plasmático del paciente con duraciones entre dos y cuatro horas, usando tasas de flujo sanguíneo al inicio de entre 50 y $80 \mathrm{ml} / \mathrm{min}$ y aumentando gradualmente hasta $100 \mathrm{o} 150 \mathrm{ml} / \mathrm{min}^{43}$.

La plasmaféresis, parece ser útil en COVID-19 debido a la afinidad entre la envoltura viral y las lectinas, sin embargo, esta modalidad requiere mayor investigación $^{43,53}$.

A partir de lo anterior, el consenso no recomienda el uso rutinario de recambio plasmático terapéutico en COVID-19 severa, sin embargo, en centros con alta experiencia, puede ser usado cuidadosamente en casos específicos evaluando riesgo-beneficio y pronóstico individual (Recomendación fuerte a favor) ${ }^{53}$.

\section{Conclusiones}

El SARS-CoV-2 y la enfermedad relacionada por COVID-19 son conocidos por la comunidad médica desde hace menos de 6 meses. Su fisiopatología es aún desconocida, sin embargo, se conoce que la 
respuesta inflamatoria sistémica y la liberación de citocinas y quimiocinas de manera excesiva, también denominado "tormenta de citocinas", se constituye como la base fundamental para entender la respuesta viral y las complicaciones sistémicas asociadas a esta enfermedad. La mayoría de las terapias son experimentales, con beneficios demostrados en el tratamiento de otras enfermedades. Sin embargo, sin contar aun con ensayos clínicos aleatorizados que confirmen su seguridad y eficacia en infección por COVID-19. La terapia de recambio plasmático se puede considerar como un tratamiento de rescate o complementario, con el fin de eliminar la tormenta de citoquinas relacionada con la insuficiencia respiratoria aguda y la disfunción multiorgánica que se presenta en la enfermedad grave, con un posible beneficio adicional de poder reducir la alta carga viral. Hace falta la realización de ensayos clínicos controlados y con buenos diseños metodológicos, que nos ayuden a demostrar la efectividad de este tipo de terapias en pacientes con COVID-19 grave.

\section{Referencias}

1. Guan W, Ni Z, Hu Y, Liang W, Ou C, He J, et al. Clinical characteristics of coronavirus disease 2019 in China. N Engl J Med. 2020; 382(18): 1708-1720. doi: 10.1056/NEJMoa2002032

2. Ksiazek TG, Erdman D, Goldsmith CS, Zaki SR, Peret T, Emery S, et al. A Novel Coronavirus Associated with Severe Acute Respiratory Syndrome. N Engl J Med. 2003; 348(20):19531966. doi: 10.1056/NEJMoa030781

3. World Health Organization. Coronavirus disease (COVID-19) outbreak (https://www .who .int). Situation report. 20 de febrero de 2020.

4. World Health Organization. Coronavirus disease 2019 (COVID-19) Situation Report -51. 11 de marzo de 2020;

5. World Health Organization. Coronavirus disease 2019 (COVID-19) Situation Report -161. 29 de junio de 2020;

6. Ministerio de Salud y Protección social, Instituto nacional de salud. Situación actual de nuevo Coronarivus (COVID-19). 29 de junio de 2020; https://d2jsqrio60m94k.cloudfront.net.

7. Lu R, Zhao X, Li J, Niu P, Yang B, Wu H, et al. Genomic characterisation and epidemiology of 2019 novel coronavirus: implications for virus origins and receptor binding. Lancet. 2020; 395(10224): 565-574. doi: 10.1016/S0140-6736(20)30251-8

8. Cong Y, Verlhac P, Reggiori F. The Interaction between Nidovirales and Autophagy Components.
Viruses. 2017; 9(7):182. doi: 10.3390/v9070182

9. Bennett JE, Dolin R, Blaser MJ. Mandell, Douglas, and Bennett's principles and practice of infectious diseases. 2020.

10. Cui J, Li F, Zheng-Li S. Origin and evolution of pathogenic coronaviruses. Nat Rev Microbiol. 2019; 17(3): 181-192. doi: 10.1038/s41579-018-0118-9

11. van Doremalen $\mathrm{N}$, Bushmaker $\mathrm{T}$, Morris $\mathrm{DH}$, Holbrook MG, Gamble A, Williamson BN, et al. Aerosol and Surface stability of SARS-CoV-2 as compared with SARS-CoV-1. N Engl J Med. 2020; 382(16): 1564-1567. doi: 10.1056/NEJMc2004973

12. Cao Z, Li T, Liang L, Wang H, Wei F, Meng S, et al. Clinical characteristics of coronavirus disease 2019 patients in Beijing, China. Feng Y-M, editor. PLOS ONE. 2020; 15(6): e0234764. doi: 10.1371/journal. pone. 0234764

13. Zhou Z, Zhao N, Shu Y, Han S, Chen B, Shu X. Effect of gastrointestinal symptoms in patients with COVID-19. Gastroenterology. 2020; 158(8): 22942297. doi: 10.1053/j.gastro.2020.03.020

14. 14. Tang Y-W, Schmitz JE, Persing DH, Stratton CW. Laboratory diagnosis of COVID-19: current issues and challenges. J Clin Microbiol. 2020; 58(6): e00512-00520. doi: 10.1128/JCM.00512-20.

15. Loeffelholz MJ, Tang Y-W. Laboratory diagnosis of emerging human coronavirus infections - the state of the art. Emerg Microbes Infect. 2020; 9(1): $747-$ 756. doi: 10.1080/22221751.2020.1745095

16. 16. Wang W, Xu Y, Gao R, Lu R, Han K, Wu G, et al. Detection of SARS-CoV-2 in different types of clinical specimens. JAMA. 2020; 323(18): 18431844. doi: 10.1001/jama.2020.3786

17. Alhazzani W, Møller MH, Arabi YM, Loeb M, Gong MN, Fan E, et al. Surviving Sepsis campaign: guidelines on the management of critically ill adults with Coronavirus disease 2019 (COVID-19). Intensive Care Med. 2020; 46(5): 854-887. doi: 10.1007/s00134-020-06022-5.

18. Maestros PS. Coronaviridae. Fields Virology Lippincott Williams y Wilkins, Philadelphia, PA. 2013; 825-858.

19. Pastrian-Soto G. Bases genéticas y moleculares del COVID-19 (SARS-CoV-2). mecanismos de patogénesis $\mathrm{y}$ de respuesta inmune. Int $\mathrm{J}$ Odontostomatol. 2020; 14(3): 331-337. doi: http:// dx.doi.org/10.4067/S0718-381X2020000300331

20. Sarzi-Puttini P, Giorgi V, Sirotti S, Marotto D, Ardizzone S, Rizzardini G, et al. COVID-19, cytokines and immunosuppression: what can we learn from severe acute respiratory syndrome? Clin Exp Rheumatol. 2020; 38(2): 337-342. 
21. Li X, Geng M, Peng Y, Meng L, Lu S. Molecular immune pathogenesis and diagnosis of COVID-19. J Pharm Anal. 2020; 10(2): 102-108. doi: 10.1016/j. jpha.2020.03.001

22. Soy M, Keser G, Atagündüz P, Tabak F, Atagündüz I, Kayhan S. Cytokine storm in COVID-19: pathogenesis and overview of anti-inflammatory agents used in treatment. Clin Rheumatol. 2020; 39(7): 2085-2094. doi: 10.1007/s10067-020-05190-5

23. Wu C, Chen X, Cai Y, Xia J, Zhou X, Xu S, et al. Risk factors associated with acute respiratory distress syndrome and death in patients with Coronavirus disease 2019 Pneumonia in Wuhan, China. JAMA Intern Med. 2020; 180(7): 1-11. doi: 10.1001/ jamainternmed.2020.0994

24. Yuen KY, Wong SSY. Human infection by avian influenza A H5N1. Hong Kong Med J. 2005;11(3): 189-199.

25. Channappanavar R, Perlman S. Pathogenic human coronavirus infections: causes and consequences of cytokine storm and immunopathology. Semin Immunopathol. 2017; 39(5): 529-539. doi: 10.1007/ s00281-017-0629-x.

26. Zhang W, Zhao Y, Zhang F, Wang Q, Li T, Liu Z, et al. The use of anti-inflammatory drugs in the treatment of people with severe coronavirus disease 2019 (COVID-19): The Perspectives of clinical immunologists from China. Clin Immunol. 2020; 214: 108393. doi: 10.1016/j.clim.2020.108393

27. Terpos E, Ntanasis-Stathopoulos I, Elalamy I, Kastritis E, Sergentanis TN, Politou M, et al. Hematological findings and complications of COVID -19. Am J Hematol. 2020; 95(7): 834-847.

28. Zhang B, Zhou X, Zhu C, Feng F, Qiu Y, Feng J, et al. Immune phenotyping based on neutrophilto-lymphocyte ratio and $\operatorname{IgG}$ predicts disease severity and outcome for patients with COVID-19. medRxiv. 2020; doi: http://medrxiv.org/lookup/ doi/10.1101/2020.03.12.20035048

29. Mehta P, McAuley DF, Brown M, Sanchez E, Tattersall RS, Manson JJ. COVID-19: consider cytokine storm syndromes and immunosuppression. Lancet. 2020; 395(10229): 1033-1034. doi: 10.1016/ S0140-6736(20)30628-0

30. Lucherini OM, Rigante D, Sota J, Fabiani C, Obici L, Cattalini M, et al. Updated overview of molecular pathways involved in the most common monogenic autoinflammatory diseases. Clin Exp Rheumatol. 2018; 36 Suppl 110(1): 3-9.

31. Crayne CB, Albeituni S, Nichols KE, Cron RQ. The Immunology of macrophage activation syndrome. Front Immunol. 2019; 10:119. doi: 10.3389/ fimmu.2019.00119
32. Daza Arnedo, R,Aroca Martínez, G,Rico Fontalvo, JE, Rey Vela E, Pájaro Galvis N, Salgado Montiel LG, et al. Terapias de purificación sanguínea en COVID-19. Rev Colomb Nefrol. 2020; 7(Supl 2).

33. Joyner M, Wright RS, Fairweather D, Senefeld J, Bruno K, Klassen S, et al. Early Safety indicators of COVID-19 convalescent plasma in 5,000 patients. medRxiv. 2020; preprint. doi: http://medrxiv.org/ lookup/doi/10.1101/2020.05.12.20099879

34. Patel P, Nandwani V, Vanchiere J, Conrad SA, Scott LK. Use of therapeutic plasma exchange as a rescue therapy in $2009 \mathrm{pH} 1 \mathrm{~N} 1$ influenza A-An associated respiratory failure and hemodynamic shock: Pediatr Crit Care Med. 2011; 12(2): e87-e89. doi: 10.1097/ PCC.0b013e3181e2a569

35. Busund R, Koukline V, Utrobin U, Nedashkovsky E. Plasmapheresis in severe sepsis and septic shock: a prospective, randomised, controlled trial. Intensive Care Med. 2002; 28(10): 1434-1439. doi: 10.1007/ s00134-002-1410-7

36. Knaup H, Stahl K, Schmidt BMW, Idowu TO, Busch M, Wiesner O, et al. Early therapeutic plasma exchange in septic shock: a prospective open-label nonrandomized pilot study focusing on safety, hemodynamics, vascular barrier function, and biologic markers. Crit Care. 2018; 22(1): 285. doi: 10.1186/s13054-018-2220-9

37. Keith P, Day M, Perkins L, Moyer L, Hewitt K, Wells A. A novel treatment approach to the novel coronavirus: an argument for the use of therapeutic plasma exchange for fulminant COVID-19. Crit Care. 2020; 24(1): 128. doi: 10.1186/s13054-0202836-4

38. Keith P, Day M, Choe C, Perkins L, Moyer L, Hays E, et al. The successful use of therapeutic plasma exchange for severe COVID-19 acute respiratory distress syndrome with multiple organ failure. SAGE Open Med Case Rep. 2020; 8: 2050313 X2093347. doi: $10.1177 / 2050313 X 20933473$

39. Shi H, Zhou C, He P, Huang S, Duan Y, Wang X, et al. Successful treatment with plasma exchange followed by intravenous immunoglobulin in a critically ill patient with COVID-19. Int J Antimicrob Agents. 2020; 105974. doi: 10.1016/j. ijantimicag.2020.105974

40. Zhang L, Zhai H, Ma S, Chen J, Gao Y. Efficacy of therapeutic plasma exchange in severe COVID-19 patients. Br J Haematol. 2020. doi: https://doi. org/10.1111/bjh.16890

41. Dogan L, Kaya D, Sarikaya T, Zengin R, Dincer A, Akinci IO, et al. Plasmapheresis treatment in COVID-19-related autoimmune meningoencephalitis: case series. Brain Behav 
Immun. 2020; 87: 155-158. doi: 10.1016/j. bbi.2020.05.022

42. Khamis F, Al-Zakwani I, Al Hashmi S, Al Dowaiki S, Al Bahrani M, Pandak N, et al. Therapeutic plasma exchange in adults with severe COVID-19 Infection. Int J Infect Dis. 2020; S1201971220304999. doi: https://doi.org/10.1016/j.ijid.2020.06.064

43. Ronco C, Reis T, De Rosa S. Coronavirus epidemic and extracorporeal therapies in intensive care: si vis pacem para bellum. Blood Purif. 2020; 49(3): 255258. doi: $10.1159 / 000507039$

44. Ronco C, Bellomo R, Kellum JA, Ricci Z. Critical care nephrology. Elsevier. Canada. 2019.

45. Padmanabhan A, Connelly-Smith L, Aqui N, Balogun RA, Klingel R, Meyer E, et al. Guidelines on the Use of therapeutic apheresis in clinical practice-evidence-based approach from the writing committee of the American Society for Apheresis: The eighth special issue. J Clin Apheresis. 2019; 34(3): 171-354. doi: 10.1002/jca.21705

46. Connelly-Smith L, Dunbar NM. The 2019 guidelines from the American Society for Apheresis: what's new? Curr Opin Hematol. 2019; 26(6): 461-465. doi: 10.1097/MOH.0000000000000534

47. Ishikawa T, Abe S, Kojima Y, Sano T, Iwanaga A, Seki K-I, et al. Prediction of a sustained viral response in chronic hepatitis $\mathrm{C}$ patients who undergo induction therapy with double filtration plasmapheresis plus interferon- $\beta /$ ribavirin. Exp Ther Med. 2015; 9(5): 1646-1650. doi: 10.3892/ etm.2015.2340

48. Zhang Y, Yu L, Tang L, Zhu M, Jin Y, Wang Z, et al. A promising anti-cytokine-storm targeted therapy for COVID-19: The artificial-liver blood-purification system. Engineering. 2020; S209580992030062X. doi: 10.1016/j.eng.2020.03.006

49. Liu X, Zhang Y, Xu X, Du W, Su K, Zhu C, et al. Evaluation of plasma exchange and continuous veno-venous hemofiltration for the treatment of severe avian influenza A (H7N9): a cohort study: blood purification in severe avian influenza A (H7N9). Ther Apher Dial. 2015; 19(2): 178-184. doi: 10.1111/1744-9987.12240

50. U.S. Authority, food and drug agency (FDA) for TORAYMYXIN $®$ to treat COVID-19 patients in a clinical study in the U.S. Regarding an approval for the interim order of "TORAYMYXIN®" to treat COVID-19 patients in Canada.

51. Adeli SH, Asghari A, Tabarraii R, Shajari R, Afshari S, Kalhor N, et al. Using therapeutic plasma exchange as a rescue therapy in CoVID-19 patients: a case series. Pol Arch Intern Med. 2020; 130(5): 455-458. doi: 10.20452/pamw. 15340
52. Kawashima H, Togashi T, Yamanaka G, Nakajima M, Nagai M, Aritaki K, et al. Efficacy of plasma exchange and methylprednisolone pulse therapy on influenza-associated encephalopathy. J Infect. 2005; 51(2): E53-56. doi: 10.1016/j.jinf.2004.08.017

53. González C, Yama E, Yomayusa M, VargasJ, Rico J, Ariza A, et al. Consenso colombiano de expertos sobre recomendaciones informadas en la evidencia para la prevención, diagnóstico y manejo de la lesión renal aguda por SARS-CoV-2/COVID-19. Rev Colomb Nefrol. 2020; 7(Supl 2). doi: https:// doi.org/10.22265/acnef.7.Suplemento.473

54. Chinese Medical Association. Expert consensus on the Application of Special Blood purification Technology in severe COVID-19 pneumonia. 